CLNS-95/1366

hep-th/9510142

October 1995

\title{
An $\mathrm{N}=2$ Dual Pair and a Phase Transition
}

\author{
Paul S. Aspinwall \\ F.R. Newman Lab. of Nuclear Studies, \\ Cornell University, \\ Ithaca, NY 14853
}

\begin{abstract}
We carefully analyze the $N=2$ dual pair of string theories in four dimensions introduced by Ferrara, Harvey, Strominger and Vafa. The analysis shows that a second discrete degree of freedom must be switched on in addition to the known "Wilson line" to achieve a non-perturbatively consistent theory. We also identify the phase transition this model undergoes into another dual pair via a process analogous to a conifold transition. This provides the first known example of a phase transition which is understood from both the type II and the heterotic string picture.
\end{abstract}




\section{Introduction}

Even though non-perturbative string theory has yet to be properly defined we have gained some insight into its structure by using the notion of duality. At this time no duality can be considered to be rigorously proven simply because this would require a full definition of non-perturbative string theory. What we can say however is that some of the many proposed dualities do not contradict what we do know of string theory and appear to be self-consistent. One might then take these reasonably well-established dualities as a defining property of string theory.

Perhaps the most powerful duality considered is that of string-string duality [1, 2]. This states that the type IIA string compactified on a K3 surface gives precisely the same sixdimensional physics as the heterotic string compactified on a 4-torus. This $N=2$ theory in six dimensions can then be compactified on a 2-torus to yield an $N=4$ theory in four dimensions giving four dimensional statements about duality as discussed in [3, [4].

Independently of this string-string duality in six dimensions, some dual pairs for $N=2$ were proposed in four dimensions in [5]. In these cases, the type IIA (or type IIB) string compactified on some Calabi-Yau manifold (or its mirror) is considered to be equivalent to the heterotic string compactified on a conformal field theory which is essentially derived from a K3 surface times a 2-torus. In [6, [] it was noted that the Calabi-Yau manifolds in question could be written as a fibration over a rational curve with generic fibre a K3 surface whereas the $\mathrm{K} 3 \times T^{2}$ case can be written as a fibration over a rational curve with generic fibre $T^{4}$. It is then tempting to try to use string-string duality in six-dimensions fibre-wise to deduce these $N=2$ dual pairs in four-dimensions. In [7] some preliminary observations were made concerning this deduction but the full picture has not yet emerged.

Another approach to obtaining more dual pairs in four dimensions is to take orbifolds of the above $N=4$ case. As we do not yet know how to deal with fixed points, it is best to start with free-actions on the type II side. There are essentially two types of free quotient of $\mathrm{K} 3 \times T^{2}$. Firstly the action can be free on the torus and have fixed points on the K3 surface. This leads to more $N=4$ theories which were studied in [8, 9, 10]. Alternatively the action is free on the K3 surface and has fixed points on $T^{2}$. This is essentially unique and was first considered in [11]. This example is the central object of study in this paper. It gives an $N=2$ theory in four dimensions but has many properties one would associate with an $N=4$ theory. In this way it is probably the most "tame" $N=2$ theory in four dimensions.

The process of orbifolding to obtain the $N=4$ theories of [8, 9, 10] was a straight-forward process. The action of the quotienting group on the K3 surface and torus on the type II side could be translated into the heterotic language to produce the dual asymmetric orbifold. In this $N=4$ case the resulting asymmetric orbifold is automatically modular invariant. We are not so lucky with the $N=2$ case of [11] however and so the orbifolding process must be more subtle if we are to obtain a dual pair. The trick appears to be to use some degrees of freedom from the R-R sector of the type II string. Such degrees of freedom are rather 
trivial from the point of view of the conformal field theory on the type II side but can effect the modular invariance properties of the conformal field theory on the heterotic side. Thus there must be some non-perturbative consistency requirement that we do not yet understand on the type II side if duality is to work. In this paper we will give a natural topological interpretation to the discrete degrees of freedom which must be employed to satisfy this non-perturbative consistency requirement. We will see that both the 1-form and the 3 -form $\mathrm{R}-\mathrm{R}$ fields in the type IIA string appear to play an important rôle.

When one considers $N=2$ theories in four dimensions the possibility of phase transitions must be considered. It was first noticed many years ago [12] that the moduli spaces of various Calabi-Yau manifolds appeared to be "connected" to each other by going through points where the 3-fold degenerates. This lead to the idea that perhaps string theories corresponding to vacua given by different Calabi-Yau manifolds could be deformed into each other [13]. The simplest kind of degeneration considered for such a process is that of a "conifold". The immediate problem with this idea was that the conformal field theory associated to the degenerate 3 -fold was itself degenerate. Thus the original idea was that string theory might "tunnel" between vacua. More recently it was realized that although the conformal field theory might be sick on the degenerate 3 -fold, the complete non-perturbative type II string was probably well-behaved [14]. In fact, an example of a "phase transition" based on a conifold concerning type II string theories compactified on different Calabi-Yau manifolds could be understood in terms of the string theory as well as algebraic geometry [15].

If there is a heterotic string theory that is dual to a given type II string on a CalabiYau manifold then one should be able to see this transition in terms of the heterotic string too. For the conifold transition studied in [15] the heterotic dual is not yet known. In [5] however, an example of a phase transition that looked a lot like a conifold transition was given. Unfortunately the type II dual for that example is not yet known. Recently some lists of potential dual pairs were given in [16] where the transitions are also understood from the heterotic side. In this paper we will describe a case where both the type IIB string picture and the heterotic string picture can be given.

It is perhaps worthwhile to spell out some nomenclature we will use here as usage of the word "conifold" has varied in the past. The term "conifold" was first introduced in [17] to refer to an algebraic variety with a finite number of nodes. A node is an isolated singularity locally of the form

$$
w^{2}+x^{2}+y^{2}+z^{2}=0 .
$$

Some later uses of the word "conifold" have been more general than this. We will use the original definition. Locally such a node can be resolved either by a deformation of complex structure, which replaces the node by a single $S^{3}$, or by a "small resolution" which replaces the node by an $S^{2}$. Within a conifold, there can be an obstruction to the small resolution if we demand that the resulting space is Kähler. If there is no such obstruction then we have a conifold transition between two Calabi-Yau manifolds. The transition and degenerations considered in this paper are not of this type. We will use the term "extremal transition" to 
refer to the general process of a topology change due to being able to resolve a singularity either by a deformation of complex structure or a deformation of Kähler form (see, for example, [18]). We will also use the term "phase transition" for this more general process. There are many extremal transitions which are not conifold transitions. An example based on a orbifold was studied in [19, 20].

The original proposal in 12 was that all Calabi-Yau manifolds should somehow be connected together by extremal transitions. It was stated in rather modest terms however. Firstly only simply-connected manifolds were considered and secondly non-Kähler manifolds were allowed as intermediate states. It was seen in 13 that a surprisingly large class of simply-connected manifolds could be connected without the need for non-Kähler manifolds. It was suggested in 15 that perhaps all $N=2$ theories in four dimensions might be connected by phase transitions. This leaves open the issue of $\pi_{1}$ however. For reasons we will give later, one might suspect that $\pi_{1}$ should not change. We will show that in our example $\pi_{1}$ can indeed change and so this potential obstacle for connecting all Calabi-Yau manifolds together is removed.

The paper is divided into two parts - one dealing with the analysis of the discrete degrees of freedom of the type II string and one dealing with the phase transition. The two parts can be read independently of each other but it is worth emphasizing that they should be considered to be intimately linked as they ultimately both analyze exactly the same vector within the lattice $\Gamma^{6,22}$. In section 2 we will analyze carefully the complete moduli space of type II strings compactified on a space in the form of $Y / G$, where $G$ acts freely. This will allow us in section 3 to identify exactly which type IIA string is dual to the heterotic string in the dual pair we consider. In section 1 we will examine closely a point in the moduli space of the type IIA and type IIB string where a rather curious degeneration occurs. In section

5 we show how this leads to the phase transition already understood from the heterotic side from [1]. Finally in section [6 we make some concluding remarks.

\section{Orbifolds Without Fixed Points}

We wish to consider orbifolds in the form $X=Y / G$ where $Y$ is a smooth manifold and $G$ is a discrete group that acts freely. Thus $X$ is also a smooth manifold. Of particular interest to us is the detailed form of the moduli space of string theories on $X$. This comes from the singular cohomology of $X$ as we now discuss. The 10-dimensional form of a string theory contains fields described by $p$-forms for various values of $p$. That is, the action will have $(p+1)$-form field-strengths. Such fields in the 10-dimensional picture can lead to moduli in the lower-dimensional theory upon compactification on $X$. The general picture is that one can "integrate" the $p$-form over some $p$-cycle within $X$. Thus the number of moduli obtained per $p$-form would appear to be $b_{p}=\operatorname{dim} H_{p}(X)$. (In general more moduli can appear from duality transformations but we need not concern ourselves with those here.)

As well as the dimension of the moduli space arising from the $p$-forms in ten-dimensional 
space-time we would also like to know the precise form of this moduli space. Given the lack of a precise definition of string theory we cannot really establish anything rigorously here but we can make some reasonable guesses. Firstly we will assert some periodicity on these moduli. This amounts to saying that shifting the moduli by an element of $H^{p}(X, \mathbb{Z})$ results in a shift in the action by $2 \pi i$ and hence has no effect on the resulting physics. This periodicity can be viewed as arising from the "quantization" of soliton charges [2] and was shown to follow directly from duality in the case of $K 3 \times T^{2}$ in [4]. Thus far we appear to be saying that the moduli space is $H^{p}(X, \mathbb{R}) / H^{p}(X, \mathbb{Z})$. There can also be extra discrete degrees of freedom coming from torsion cycles within $X$. That is, we may have a singular cycle, $C$, which is homologically non-trivial but $n[C]=0$ for some integer $n$. We can consistently attach phases to such cycles to give discrete degrees of freedom to the moduli. This extra degree of freedom is not particularly new as in the case $p=1$ they correspond to nothing more than "Wilson lines". The case $p=2$ has already been considered in string theory for the $B$-field in the context of "discrete torsion" [21].

All said, we claim that each $p$-form in the ten-dimensional picture contributes a factor

$$
\begin{aligned}
\mathscr{M}_{p} & =U(1)^{b_{p}} \times \operatorname{Tors}\left(H_{p}(X)\right) \\
& =U(1)^{b_{p}} \times \operatorname{Tors}\left(H^{p+1}(X)\right) \\
& =H^{p}(X, U(1)) \\
& =\operatorname{Hom}\left(H_{p}(X), U(1)\right)
\end{aligned}
$$

to the moduli space, where Tors denotes the torsion part. See [22 for the methods used to obtain the various equalities in (2). Our notation will always assume (co)homology to be over the integers unless otherwise specified. In the case of $p=1$ we have $\mathscr{M}_{1}=$ $\operatorname{Hom}\left(H_{1}(X), U(1)\right)=\operatorname{Hom}\left(\pi_{1}(X), U(1)\right)$. This is precisely the moduli space of flat $U(1)$ bundles on $X$. For $p>1$ we are making a similar statement for "currents" arising from $(p-1)$-branes. In particular, as stated above, we have already encountered $\mathscr{M}_{2}$ in string theory [21, 23].

Now we want to determine the moduli space for the orbifold $X=Y / G$. Locally it is certainly the case that the moduli of $X$ are the $G$-invariant moduli of $Y$. Thus, we first need to establish the moduli space of $Y$. Then we should consider the possibility that there may be homology cycles in $X$ that did not descend from $Y$. That is, the quotient map $f: Y \rightarrow X$ yields

$$
f_{*}: H_{*}(Y)^{G} \rightarrow H_{*}(X)
$$

which is injective but not an isomorphism. $G$ as a superscript denotes the $G$-invariant part. The cokernel of this map gives us precisely the extra discrete degrees of freedom present in the orbifold. In this paper we will work with cohomology as well as homology. The reader is referred to [22] for the detailed relationship between these groups. 
Our main weapon for comparing the cohomology of $X$ and $Y$ is the Hochschild-Serre spectral sequence [24]. That is, there is a spectral sequence with second stage

$$
E_{2}^{p, q}=H^{p}\left(G, H^{q}(Y)\right)
$$

This converges to $E_{\infty}^{p, q}$ where

$$
H^{n}(X)=\bigoplus_{p+q=n} E_{\infty}^{p, q}
$$

Detailed analysis of this spectral sequence can be rather messy in general but we can make some simple statements. Firstly the term $E_{2}^{p+1,0}=H^{p+1}(G)$ is simple group cohomology (that is, of the form $H^{*}(G, \mathbb{Z})$ where $G$ acts trivially on $\mathbb{Z}$ ). Unless killed by earlier stages of the spectral sequence this will have either of two effects on $H^{n}(X)$. Firstly it can remove some of $E_{2}^{0, p} \cong H^{p}(Y)^{G}$ from $H^{p}(X)$. Secondly it can contribute torsion to $H^{p+1}(X)$. Either of these effects will increase the discrete degrees of freedom in $\mathscr{M}_{p}$ relative to the moduli space of $Y$. If we assume $Y$ is simply connected and $G$ is finite then for the case $\mathscr{M}_{1}$ these new discrete degrees of freedom are in fact given precisely by $H^{2}(G)$, which is the abelianization of $G$, and for the case $\mathscr{M}_{2}$ we have $H^{3}(G) \cong H^{2}(G, U(1))$. This simplification explains why we have not had to suffer the complexities of spectral sequences in the past. For $p=1$, the new degrees of freedom, "Wilson Lines" were given simply by the quotienting group itself and for $p=2$ we only had to worry about basic group cohomology to find the "discrete torsion". In the cases $p>2$ life appears to demand the Hochschild-Serre spectral sequence in its full glory to understand the effect of orbifolding.

In general other entries in $E_{2}^{p, q}$ will also effect these discrete degrees of freedom. In particular, one of the contributions can come from the fact that the moduli space of $G$ invariant theories on $Y$ is not connected even though the complete moduli space of theories on $Y$ is connected.

Let $Y$ be a product of a $\mathrm{K} 3$ surface and a 2-torus as in [11. $Y$ admits a symmetry $G \cong \mathbb{Z}_{2}$. The generator, $g$, of $G$ acts freely on the K3 surface. The quotient K3/G is known as the "Enriques Surface" and is not a Calabi-Yau surface. In particular, if $\omega$ is a $(2,0)$-form on the K3 surface then

$$
g: \omega \rightarrow-\omega .
$$

If the 2-torus is represented by periodic identification of the 2-plane with coordinates $(x, y)$ then $g:(x, y) \rightarrow(-x,-y)$. Thus $g$ has 4 fixed points on $T^{2}$. The complete action of $g$ is free as required.

The topology of $X$ is very rich and is one of the reasons why a full understanding of string duality on this manifold would provide great insight into the general case. The global holonomy of $X$ is $S U(2) \times \mathbb{Z}_{2}$. Thus, although $Y$ is a Calabi-Yau manifold it does not have the generic holonomy $S U(3)$. This leads to some simplifications such as no world-sheet instanton corrections to the type II string. 


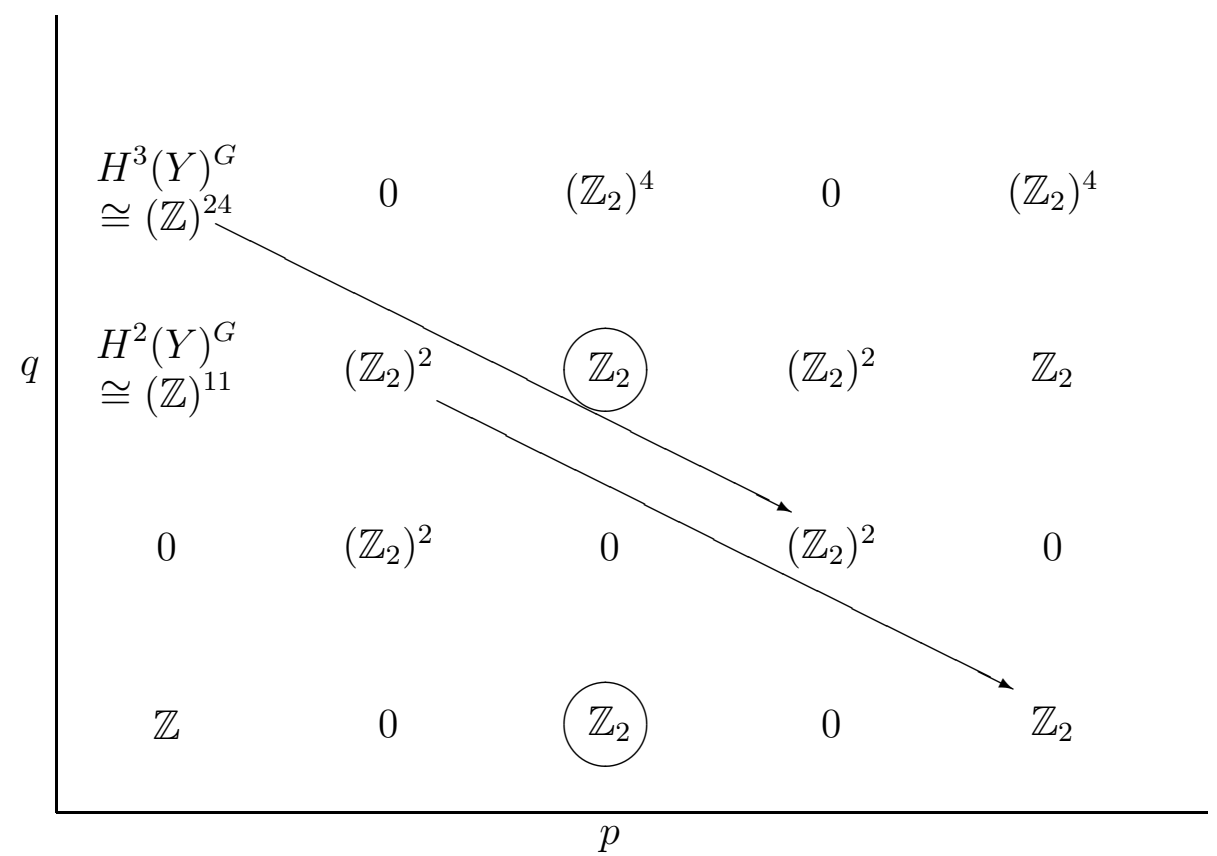

Figure 1: Hochschild-Serre spectral sequence term for $E_{2}^{p, q}$.

Next consider the fundamental group. If $\bar{X}$ is the simply-connected covering space of $X$ then $\bar{X} / \pi_{1}(X) \cong X$. Thus

$$
\pi_{1}(X) \cong \mathbb{Z}_{2} \ltimes(\mathbb{Z})^{2},
$$

where the $\mathbb{Z}_{2}$ acts on the lattice $(\mathbb{Z})^{2}$ as $g$ acted on the torus.

We can now give the cohomology for the case in question from the above discussion. In terms of homology, which is clearer for our purposes,

$$
\begin{aligned}
& H_{0}(X)=\mathbb{Z} \\
& H_{1}(X)=\left(\mathbb{Z}_{2}\right)^{3} \\
& H_{2}(X)=(\mathbb{Z})^{11} \times \mathbb{Z}_{2}, \quad H_{2}(X) /\left(f_{*} H_{2}(Y)^{G}\right)=\mathbb{Z}_{2} \\
& H_{3}(X)=(\mathbb{Z})^{24} \times \mathbb{Z}_{2}, \quad H_{3}(X) /\left(f_{*} H_{3}(Y)^{G}\right)=\left(\mathbb{Z}_{2}\right)^{3} \\
& H_{4}(X)=(\mathbb{Z})^{11} \times\left(\mathbb{Z}_{2}\right)^{3} \\
& H_{5}(X)=0 \\
& H_{6}(X)=\mathbb{Z} .
\end{aligned}
$$

This is obtained from the spectral sequence shown in figure 11. To compute the exact progression of this spectral sequence we need to compare this spectral sequence to that for the Enriques surface as quotient of a K3 surface. We also need to know that $X$ can be written 
as an elliptic fibration over an Enriques surface and that this bundle has a section. One can then show that $H^{3}(X)$ has torsion as given in (8) and that the two $d_{3}$ maps shown in figure 1 are surjective. Note that we recover the result $h^{1,1}(X)=h^{2,1}(X)=11$.

Now let us consider compactifying the type IIA superstring on the Calabi-Yau manifold $X$. In order to analyze the moduli space we first need the (connected part of) the moduli space of a IIA string on $Y$ that admits $G$ as a symmetry. This is readily shown to be [11]

$$
\mathscr{M}_{Y}^{G} \cong \mathscr{M}_{Y}^{V} \times \mathscr{M}_{Y}^{H}
$$

where

$$
\begin{aligned}
& \mathscr{M}_{Y}^{V} \cong S l(2, \mathbb{Z}) \backslash S l(2) / U(1) \times O\left(\Gamma^{2,10}\right) \backslash O(2,10) /(O(2) \times O(10)) \\
& \mathscr{M}_{Y}^{H} \cong O\left(\Gamma^{4,12}\right) \backslash O(4,12) /(O(4) \times O(12)),
\end{aligned}
$$

where $\Gamma^{p, q}$ is a lattice with intersection form signature $(p, q)$ and $O\left(\Gamma^{p, q}\right)$ is its group of rotational isometries. Note that neither $\Gamma^{2,10}$ nor $\Gamma^{4,12}$ in (10) are self-dual.

From (8) we can now find the extra discrete degrees of freedom exhibited by the type IIA string on $X$. Firstly the NS-NS sector of the type IIA string has a 2-form. This gives a $\mathbb{Z}_{2}$-valued parameter. The NS-NS degrees of freedom should be visible from the conformal field theory. This is not really "discrete torsion" in the sense of 21 as $H^{3}\left(\mathbb{Z}_{2}\right)=0$ but rather has its origins in the fact that the moduli space of $G$-invariant theories on $Y$ is not connected. We will not discuss this further although it would be interesting to analyze this in terms of mirror symmetry and the consequences for the heterotic dual picture.

In the $\mathrm{R}$ - $\mathrm{R}$ sector we have a 1 -form and a 3 -form. First take the 1 -form. The first homology of $X$ is entirely torsion in the form $\left(\mathbb{Z}_{2}\right)^{3}$. This gives us effectively a choice of 7 non-trivial Wilson lines. Two of the $\mathbb{Z}_{2}$ 's may be considered to be remnants of the first homology of the $T^{2}$. The other $\mathbb{Z}_{2}$ corresponds to usual picture of lines in $Y$ whose ends are identified by $G$ descending to closed loops in $X$.

The 3-form also contributes non-trivial choices. In this case the extra homology is of the form $\left(\mathbb{Z}_{2}\right)^{3}$ where one of these factors appears as torsion in $H_{3}$. Again two of these factors are remnants of the 1-cycles on the torus. The torsion term comes from $E_{2}^{2,2}=$ $H^{2}(Y)^{G} /\left((1+g) H^{2}(Y)\right)$. This also has origins in the torus - it arises from the class in $H^{2}(Y)$ which is dual to the homology class of the torus itself.

\section{The Heterotic Dual}

If we accept that the type IIA string compactified on a K3 surface is dual to the heterotic string on $T^{4}$ then it follows that the type IIA string on $Y$ is dual to a heterotic string on $T^{6}$. One can then try to orbifold this dual pair by our $\mathbb{Z}_{2}$-action to form another dual pair with $N=2$ supersymmetry in four dimensions. Making this orbifolding process nonperturbatively correct is not a simple matter however [11, 7]. 
First let us interpret the moduli space given in (91). The moduli space of type IIA strings on $Y$ is given by [2, 国]

$$
\mathscr{M}_{Y}=O\left(\Gamma^{6,22}\right) \backslash O(6,22) /(O(6) \times O(22)) \times S l(2, \mathbb{Z}) \backslash S l(2) / U(1) .
$$

In this case $\Gamma^{6,22}$ is an even self-dual lattice that can be identified as $\Gamma^{6,22}=\Gamma^{4,20} \oplus \Gamma^{2,2}$, where $\Gamma^{4,20} \cong H^{*}(\mathrm{~K} 3)$ [25] and $\Gamma^{2,2}$ is the Narain lattice $H^{1}\left(T^{2}\right) \oplus H_{1}\left(T^{2}\right)$ as in [26].

Given that $\Gamma^{6,22}$ can also be identified as the Narain lattice for compactification of the heterotic string we now have an idea of the identification between these dual pairs. In particular, as we know how $G$ acts on the cohomology of $Y$, we can almost see how it acts on the Narain lattice. Unfortunately our knowledge is not complete. What we find by this route is only how $G$ acts by rotations on the Narain lattice. We also need to know about translations.

In order to understand the translations we look at the gauge group of the string theory. On the heterotic side, for a generic torus, the gauge group arises from the metric à la KaluzaKlein from isometries of the torus. This corresponds to the translations of the Narain lattice and the gauge group is generically $U(1)^{28}$. How does this $U(1)^{28}$ arise on the type IIA side and how does this fit into the type II interpretation of $\Gamma^{6,22}$ ?

Firstly we have a metric in the ten-dimensional theory which produces gauge fields from isometries, i.e., $H^{1}(Y)$. Secondly we have a 2-form which compactifies on 1-cycles $H_{1}(Y)$ to give gauge fields. These two contributions obviously come from the $\Gamma^{2,2}$ of $T^{2}$. Next we have a 1-form in the ten-dimensional theory which descends trivially down into a $U(1)$ gauge group upon compactification. It seems natural to identify this with the $H^{0}(\mathrm{~K} 3)$ direction. We also have a 3-form which compactifies on 2-cycles to give gauge fields. 22 of these 2-cycles come from $H_{2}(\mathrm{~K} 3) \cong H^{2}(\mathrm{~K} 3)$. The last gauge field comes from the 2-cycle given by $T^{2}$ itself. Within $Y$ this homology element maps to $H^{4}(\mathrm{~K} 3)$ under the isomorphism $H_{2}(Y) \cong H^{4}(Y)$. This completes our identification of all 28 gauge fields with shifts in $\Gamma^{6,22}$.

If we ignore translations and take the asymmetric orbifold of the heterotic string by the rotation of $\Gamma^{6,22}$ induced by $G$, it was observed in 11 that one could not obtain a modular invariant theory because of level-matching problems [27]. If $g$ acts by both a rotation and a shift simultaneously however, one can satisfy level-matching.

The shift used in [11] was uniquely identified as a half-shift along the lattice vector of length (squared) -2 contained in the lattice generated by $H^{0}(\mathrm{~K} 3)$ and $H^{4}(\mathrm{~K} 3)$. That is, both $H^{0}(\mathrm{~K} 3)$ and $H^{4}(\mathrm{~K} 3)$ must be shifted as each of these directions alone has length zero. The ability to shift by this half-vector of the lattice must manifest itself as one of the discrete degrees of freedom of the orbifold theory on $X$. Let us try to find exactly which degree of freedom it should be.

Since the $H^{0}(\mathrm{~K} 3)$ direction comes from the 1-form in ten-dimensions, this must be associated with an element of $H_{1}(X)$. The new element of $H_{1}(X)$ introduced in the quotienting process was the "Wilson line" joining points in $Y$ identified by $G$. Thus, this shift in $H^{0}(\mathrm{~K} 3)$ 
corresponds to "switching on" this "Wilson line" degree of freedom in the orbifold $X$. This was also observed in [11].

The $H^{4}(\mathrm{~K} 3)$ direction comes from the 3-form in ten-dimensions compactified over the $T^{2} \subset Y$. As explained above, a new element in $H_{3}(X)$ arises from this torus from the $E_{2}^{2,2}$ term in the spectral sequence. It would thus appear that we also have to switch on this discrete degree of freedom too to obtain a truly consistent non-perturbative string theory.

To summarize, the R-R fields in the 10-dimensional type II string theory give discrete parameters in the compactified theory. Modular invariance of the dual heterotic string theory demands that some of these parameters are "switched on". One is associated with 1-cycles on $X$ and one is associated with 3-cycles on $X$. Both of these cycles are torsion elements of the homology of $X$. The origins of these discrete degrees of freedom from the spectral sequence are circled in figure 1 .

\section{Degenerations}

Consider the moduli space of type II string theories on $\mathrm{K} 3 \times T^{2}$. Can we undergo a phase transition to another class of theories? That is, are there any extremal transitions of $\mathrm{K} 3 \times T^{2}$ ? The answer is no. To undergo an extremal transition one first goes to the boundary of moduli space a finite distance away where something degenerates. This we can do by letting the K3 surface acquire an orbifold singularity. We then try to resolve this singularity in a way inequivalent to how we reached the degeneration. This we cannot do - any attempt to resolve the orbifold singularity will return us to $\mathrm{K} 3 \times T^{2}$. Thus, type II strings on $\mathrm{K} 3 \times T^{2}$ appear to be an isolated island in the grand moduli space of all theories. The same is also true of each of the other $N=4$ quotients of [8, 9, [10].

Can we expect anything different for $X=\left(\mathrm{K} 3 \times T^{2}\right) / \mathbb{Z}_{2}$ then? In [11] it was argued from the heterotic side that there was a point in a given connected component of the moduli space of these theories where a phase transition was possible. In this section we will argue the same from the type II side.

How can $X$ acquire a singularity? There are two ways. Firstly the covering space $Y$ may be singular. From what we said above however, such a degeneration cannot be interesting from the point of view of phase transitions as all we can do to move away from such a point is to go back into the moduli space from whence we came. Alternatively the quotienting process by $G$ may degenerate. That is, $G$ is meant to act freely but at the boundary of the moduli space it can acquire fixed points. This latter case offers a doorway into other moduli spaces as we shall see.

The moduli space of type II strings on $Y$ was given in (11). The first factor is the only one of interest to us and may be regarded as quotient of the Grassmanian of space-like 6-planes in $\mathbb{R}^{6,22}$ by the isometry group of the even self-dual lattice $\Gamma^{6,22} \subset \mathbb{R}^{6,22}$. The connected component of the moduli space of $G$-invariant theories given in (9) is obtained easily from

this by analyzing how $G$ acts on the lattice $\Gamma^{6,22}$ and the 6-plane. From our knowledge of 
how to interpret $\Gamma^{6,22}$ in terms of $Y$ from section 3 we can decompose $\Gamma^{6,22}$ as

$$
\Gamma^{6,22}=\Gamma_{1}^{1,9} \oplus \Gamma_{2}^{1,9} \oplus \Gamma_{1}^{1,1} \oplus \Gamma_{2}^{1,1} \oplus \Gamma^{2,2},
$$

where each of the above terms on the right are even self-dual, and then identify the action of $G$ as generated by

$$
g:\left(\Gamma_{1}^{1,9}, \Gamma_{2}^{1,9}, \Gamma_{1}^{1,1}, \Gamma_{2}^{1,1}, \Gamma^{2,2}\right) \mapsto\left(\Gamma_{2}^{1,9}, \Gamma_{1}^{1,9}, \Gamma_{1}^{1,1},-\Gamma_{2}^{1,1},-\Gamma^{2,2}\right) .
$$

The result given in (10) is derived given that $\Gamma^{2,10} \subset \Gamma^{6,22}$ is the $G$-invariant part of the lattice and $\Gamma^{4,12} \subset \Gamma^{6,22}$ is the set of lattice vectors orthogonal to $\Gamma^{2,10}$. The 6 -plane itself, which we will call $\Pi_{6}$, must be the product of a 2-plane, $\Pi_{2}$, which is invariant under $G$ and a 4-plane, $\Pi_{4}$, such that any vector in $\Pi_{4}$ is inverted by $g$. The result is that the moduli space is a quotient of the product of the Grassmanian of space-like 2-planes in $\mathbb{R}^{2,10} \supset \Gamma^{2,10}$ and the Grassmanian of space-like 4-planes in $\mathbb{R}^{4,12} \supset \Gamma^{4,12}$. The former is a factor in the moduli space generated by the scalars coming from vector multiplets and the latter forms the moduli space generated by the hypermultiplets.

The lattice $\Gamma^{2,10}$ is naturally interpreted as the lattice of invariant cohomology on the original K3 surface. This lattice contains the Picard lattice of the K3 surface. The space, $\mathscr{M}_{Y}^{N}$ of (10), associated with this is therefore identified with moduli space of Kähler forms and $B$-fields on $X$. Note that the moduli space of Kähler forms and $B$-fields on the original $T^{2}$ gives a separate $S l(2, \mathbb{Z}) \backslash S l(2) / U(1)$ factor. The space $\mathscr{M}_{Y}^{H}$ comes from the moduli space of complex structures on $X$, the R-R moduli and the axion-dilaton system. $\mathscr{M}_{Y}^{H}$ has a quaternionic structure.

For reasons explained in [1, 28] we expect interesting things to happen in a type II string theory on $Y$ whenever the space-like 6-plane is orthogonal to elements of $\Gamma^{6,22}$ of length -2 . In particular the set of such elements should give the root diagram of a semi-simple group which will be the non-abelian part of the gauge group of the string theory. The underlying conformal field theory is expected to degenerate at such a point [28, 29]. This is therefore a prime candidate for a point at the edge of moduli space where we might expect a phase transition to live.

Consider an element, $e_{1} \in \Gamma_{1}^{1,9}$, of (13) of length -2 . Let us try to make this orthogonal to the 6 -plane, $\Pi_{6}$. Let $e_{2}=g\left(e_{1}\right)$, that is $e_{2}$ is a vector of length -2 in $\Gamma_{2}^{1,9}$. We see that $e_{1}+e_{2} \in \Gamma^{2,10}$ and $e_{1}-e_{2} \in \Gamma^{4,12}$. If we arrange $\Pi_{2}$ so that it is orthogonal to $e_{1}+e_{2}$ and $\Pi_{4}$ so that it is orthogonal to $e_{1}-e_{2}$ then we will have $\Pi_{6}$ orthogonal to $e_{1}$ as desired. We also necessarily have $e_{2}$ orthogonal to $\Pi_{6}$ at the same time. We can identify these two vectors with $S^{2}$ 's within the $\mathrm{K} 3$ surface in $Y$ as explained in [28]. When we move $\Pi_{2}$ and $\Pi_{4}$ to be orthogonal to these vectors we cause these spheres to shrink down to zero size. We also fix the $B$-field in the direction associated with the homology classes of these spheres and we have also fixed the $\mathrm{R}-\mathrm{R}$ moduli to have zero values in these directions.

Thus the situation is very similar to that of type IIA strings on a K3 surface. At particular places in the moduli space we obtain an enhanced gauge symmetry. This is associated with 
2-spheres shrinking down to zero size. For the IIA string on a K3 surface we had to impose a vanishing condition on the $B$-field and in the above case we also have to impose a condition on the R-R moduli.

The effect of quotienting by $G$ is to identify the two $S^{2}$ 's given by $e_{1}$ and $e_{2}$. Thus we only get generically a single $S U(2)$ as the gauge group on $X$. The conformal field theory associated to the heterotic string picture of this theory will have the associated affine algebra at level 2 however. By applying the same procedure to further length -2 vectors we may enhance the gauge group beyond $S U(2)$ in the usual way.

What we have just described is the process of $Y$ becoming singular because the covering space $X$ has acquired singularities. Not surprisingly this turns out to locally look like a dimensional reduction of the type IIA string on a K3 surface case. That is, in addition to the vector multiplet becoming massless we also have a hypermultiplet in the same representation of the gauge group [11. We can also see this directly from the heterotic picture. In this case we obtain massless states due to winding/momentum modes around $\pm e_{1}$ and $\pm e_{2}$. The fact that we can form both invariant and anti-invariant vectors from $e_{1}$ and $e_{2}$ shows that we get a hypermultiplet and a vector multiplet from each. This is like the $N=4$ Yang-Mills theory of [30]. There are no phase transitions associated to this as we expected.

In order to achieve this degeneration we needed to choose a special point within $\mathscr{M}_{V}$ and $\mathscr{M}_{H}$ at the same time. That is, both the vector multiplet and hypermultiplet moduli had to be tuned simultaneously. If we want to obtain a degeneration by tuning just one of the sets of moduli we need to look for length -2 vectors within $\Gamma^{2,10}$ or $\Gamma^{4,12}$. Let us focus on the vector multiplet moduli space, i.e., look for a vector of length -2 within $\Gamma^{2,10}$. There are only two such vectors which we denote $e_{F}$ and $-e_{F}$. Thus, if $\Pi_{2}$ is perpendicular to $e_{F}$ then we have an enhanced gauge group $S U(2)$ again. What is the geometrical interpretation of this?

We have identified $\Gamma^{2,10}$ as the invariant part of the total cohomology of the K3 surface. The only vectors of length -2 in this lattice are $\pm\left(e_{0}-e_{4}\right)$ where $e_{0}$ generates $H^{0}(\mathrm{~K} 3)$ and $e_{4}$ generates $H^{4}(\mathrm{~K} 3)$ - i.e., the same direction as we considered in section 3! When we use the results of [25, 31] to interpret a point in the moduli space where $\Pi_{2}$ is orthogonal to $e_{f}$, we deduce that the K3 surface has volume of order the Planck scale. That is, it is the effects of quantum geometry on the K3 surface that induces the enhanced gauge group. We can usually understand quantum geometry in terms of classical geometry by using mirror symmetry and this is no exception.

The mirror of a type IIA string theory compactified on $X \cong\left(\mathrm{K} 3 \times T^{2}\right) / G$ is a type IIB string compactified on another space $X^{\prime} \cong\left(\mathrm{K} 3 \times T^{2}\right) / G . X$ and $X^{\prime}$ are a mirror pair and are topologically the same. The moduli of $X$ and $X^{\prime}$ are not generically the same however. Let us analyze the type IIB string compactified on $X^{\prime}$.

To solve a notational problem, rather than considering a type IIB theory compactified on $X^{\prime}$, from now on we will consider the type IIB theory to be compactified on $X$. This means that $\Gamma^{2,10}$ is now anti-invariant under $g$ and $\Gamma^{4,12}$ is invariant in the type IIB context. In 

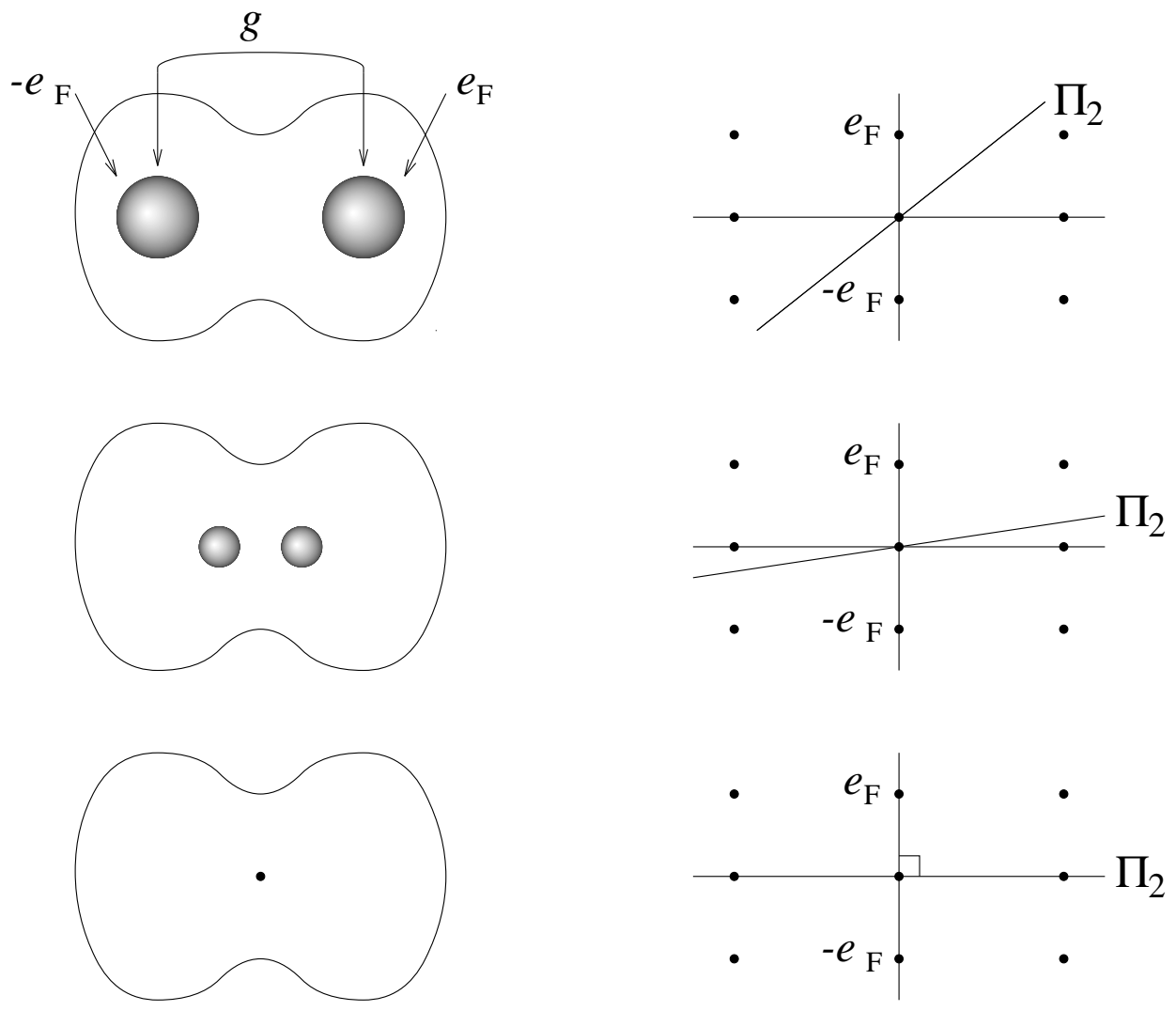

Figure 2: The degeneration of the K3 surface for $e_{F}$.

particular, $\mathscr{M}_{Y}^{N}$ is now the moduli space of complex structures on $X$ and $\mathscr{M}_{Y}^{H}$ is the moduli space of "quaternionified" Kähler forms. Thus, rather than requiring $X$ to be a particular size to get our $S U(2)$ gauge group in the type IIA case, we can ask that $X$ takes a particular complex structure in the type IIB case.

For type IIB strings, the lattice $\Gamma^{2,10}$ is now identified with the lattice orthogonal to the invariant sublattice of $H^{*}(\mathrm{~K} 3)$. This is a subspace of $H^{2}(\mathrm{~K} 3)$. We can view $\Pi_{2}$ as the 2-plane in $H^{2}(\mathrm{~K} 3, \mathbb{R})$ which is spanned by the real and imaginary parts of the holomorphic 2-form, $\omega$, on the K3 surface.

Let us suppose first that $\Pi_{2}$ is not perpendicular to $e_{F}$. We have an $S^{2}$ within the K3 surface whose homology class is given by $e_{F}$. As $g$ acts freely on the K3 surface there must be another sphere in the K3 surface which is the image of the first sphere under $g$ and whose homology class is $-e_{F}$. See figure 2 for this configuration. Now rotate $\Pi_{2}$ to be perpendicular to $e_{F}$. Now these spheres should become rational curves as they are dual to $(1,1)$-forms. This cannot be however since this would imply that the curves given by $e_{F}$ and $-e_{F}$ are both "effective" which is impossible (see, for example, 32]). Thus something must 
have gone wrong. That is to say, we simply cannot build an Enriques surface as the free quotient of a K3 surface if the K3 surface has this special complex structure. Nothing has gone wrong with the K3 surface itself so far as its complex structure is concerned so it must be the quotienting process which has degenerated.

The way to picture the situation is shown in figure 2. When $e_{F}$ is orthogonal to $\Pi_{2}$, it becomes a rational curve at which point its area is given by integrating the Kähler form over it. In this case, the Kähler form is invariant under $G$ and so must be orthogonal to $e_{F}$. Thus the areas of both spheres are zero. This is not all that happens however. In order to resolve the paradox above these spheres must become a fixed point of the quotienting. That is to say, as we rotate $\Pi_{2}$ to become perpendicular to $e_{F}$, the two spheres in the K3 surface both shrink down to zero size and coalesce. Taking the orbifold of this K3 surface by this $\mathbb{Z}_{2}$ action will result in a degenerate Enriques surface. This surface is quite interesting and has been analyzed in many places such as [33, 34]. In our case, the threefold $X$ will degenerate to singular object which we call $X^{\sharp}$.

Now let us review the heterotic side of this story given in [11]. The winding/momentum modes around $\pm e_{F}$ again give massless modes. As $\pm e_{F}$ are invariant under $g$ we get only vector multiplets from these lattice elements. The gauge group has been enhanced to $S U(2)$. In addition to these massless states we obtain more from the twisted sector. The energy of a $g$-twisted left-moving string of length $-l^{2}$ with no oscillators is

$$
\begin{aligned}
E_{L}^{g} & =\frac{1}{4} \sum_{i=1}^{22} g_{i}\left(1-g_{i}\right)-1+\frac{1}{2} l^{2} \\
& =\frac{1}{2} l^{2}-\frac{1}{4},
\end{aligned}
$$

where the eigenvalues of the rotation of the lattice are given by $\exp \left(2 \pi i . g_{i}\right)$.

Recall that in section 03 we made $g$ act by a translation on the lattice $\Gamma^{6,22}$ by $\frac{1}{2} e_{F}$. Thus, the shortest strings in the twisted sector are those given by the vectors $\pm \frac{1}{2} e_{F}$ and these have zero energy. That is, they are massless states. This zero energy-level was precisely the reason why this translation was introduced in the first place - in order to satisfy level-matching. Under $g$, these lattice vectors are anti-invariant and so these new states in the heterotic string are hypermultiplets. They also have half the $U(1)$-charge of the vector multiplets which became massless to give the $S U(2)$ gauge group. It follows that they are 2 's of $S U(2)$. Following methods from [27] one can count the degeneracy and finds one gets 4 states. All counted therefore, at this point corresponding to the type IIB string on $X^{\sharp}$ we obtain an enhanced gauge group of $S U(2)$ with four hypermultiplets in the fundamental representation becoming massless. This situation was considered in [30] where it played a special rôle due to the fact it is conformally invariant. Conformal invariance was important for consistency of the duality picture too as explained in [11].

We now see a peculiar connection between this section and section 3. The translation by $\frac{1}{2} e_{F}$ that was allowed by discrete degrees of freedom coming from the R-R sector of the type 
II string plays an essential rôle in finding the massless spectrum of the theory at the most interesting point in the moduli space of theories.

\section{A Phase Transition}

In [15] an example of a phase transition was given. This example consisted of a conifold transition in which one degenerates the complex structure of the starting manifold to acquire a collection of isolated "nodes" each of which is locally of the form

$$
w^{2}+x^{2}+y^{2}+z^{2}=0
$$

in $\mathbb{C}^{4}$ with coordinates $(w, x, y, z)$. If this is done in just the right way then the resulting singular space can be blown-up by a small resolution (replacing points by $\mathbb{P}^{1}$ 's) into a new manifold.

Since (15) is one of simplest singularities one obtains in three dimensions it is reasonable to expect conifold transitions to be fairly common. There is another singularity which is just as simple: namely

$$
x^{2}+y^{2}+z^{2}=0
$$

In this case, the singularity occurs for all $w$ and so occurs along a whole object of complex dimension one within the threefold. That is, (15) is a singularity of codimension three whereas (16) is codimension two. Codimension two singularities can appear in K3 surfaces and have been considered in [1, 28]. In the K3 case one can obtain enhanced non-abelian gauge groups when such singularities appear and then (16) gives $S U(2)$. It therefore seems not unreasonable to expect the same thing to happen for threefolds. Note that there is no reason to expect enhanced gauge groups for conifold points, i.e., codimension three singularities. In fact, an example of the heterotic dual picture of a conifold transition was conjectured in [5] and no enhancement occurs.

In our case $X^{\sharp}$ contains a whole $\mathbb{P}^{1}$ of singularities. At a generic point on this curve the singularity is of the form (16). It should not come as a surprise then to get an enhanced $S U(2)$ at this point in the moduli space of $X$ 's.

At four points on the $\mathbb{P}^{1}$ of singularities the singularity is worse than (16). These singularities are related to degenerate Enriques surfaces of the form discussed in section 4 .

Now let us try to resolve $X^{\sharp}$ to obtain a new manifold. Consider first a $\mathbb{Z}_{2}$-action on a K3 surface which does not act freely but rather fixes a rational curve. Consider the limit of the resulting quotient as we blow this fixed rational curve down to a point. It is not hard to convince oneself that the resulting space is essentially the same degenerate Enriques surface we had above. Taking this up to three complex dimensions it is clear how to proceed. Let $Y=\mathrm{K} 3 \times T^{2}$ and consider a group $G^{\prime} \cong \mathbb{Z}_{2}$ generated by $g^{\prime}$ which acts as a symmetry of

\footnotetext{
${ }^{1}$ In this case the gauge group contains a nonabelian factor of $E_{6}$ but this plays no essential rôle in the transition. It is present both before and after the conifold transition.
} 


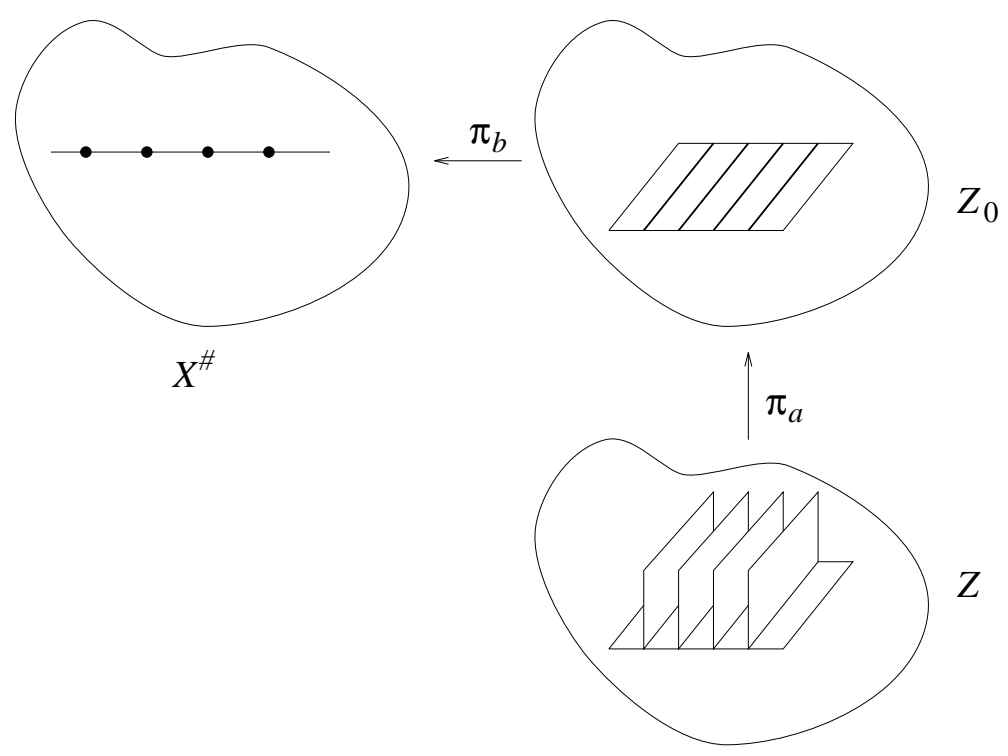

Figure 3: Blowing up $X^{\sharp}$ into $Z$.

$Y . g^{\prime}$ is similar to $g$ in that it has the same action on $T^{2}$ and satisfies (6). The difference is that $g^{\prime}$ fixes a rational curve, $C$, within the K3 surface. Let $Z_{0}=Y / G^{\prime}$.

The space $Z_{0}$ is itself singular as the orbifold had fixed points. We can blow $Z_{0}$ up to obtain $Z$ which is smooth. On the other hand, by varying the Kähler form on $Z_{0}$ we can change the area of $C$. In the limit that this area goes to zero we claim that $Z_{0}$ becomes the same space as $X^{\sharp}$. This shows that $X$ can be deformed into $Z$ by going via the singular space $X^{\sharp}$ as

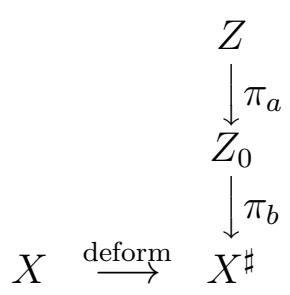

The maps $\pi_{a}$ and $\pi_{b}$ are both blow-downs, i.e., deformations of Kähler form and the horizontal map is a deformation of complex structure.

The Hodge numbers of $Z$ are simple to compute. The existence of a rational curve to be fixed on the K3 surface will obstruct one deformation of complex structure relative to $X$. Thus $h^{2,1}(Z)=10$. The orbifold $Z_{0}$ has 4 fixed $\mathbb{P}^{1}$ 's. Blowing these up gives a contribution of 4 to $h^{1,1}$ from $\pi_{a}$. $\pi_{b}$ blows just one curve down and so contributes 1 to $h^{1,1}$. Thus, $h^{1,1}(Z)=11+4+1=16$. One can also show that $Z$ is simply-connected. We show the blow-up schematically in figure 3 by showing complex dimensions as real.

The heterotic story of this transition was given in [11]. We degenerate the original theory 


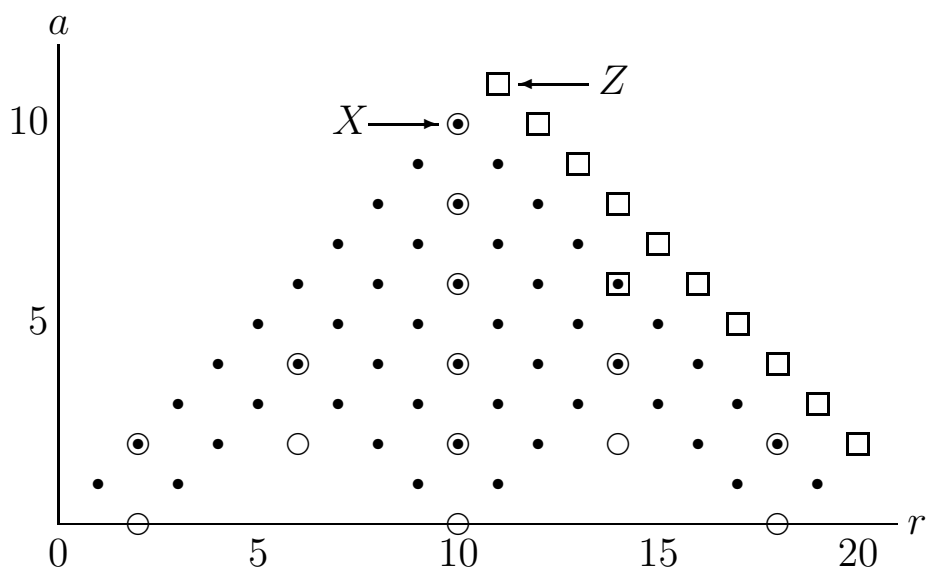

Figure 4: Voisin's Calabi-Yau manifolds. ( $X$ is the circle.)

in the "Coulomb" phase by varying a scalar from a vector multiplet to enhance one of the $U(1)$ factors in the gauge group to $S U(2)$. At this point four hypermultiplets in the form of 2's of this $S U(2)$ become massless. By giving non-zero values to the scalars in these new hypermultiplets we can venture off into the "Higgs" phase. The number of extra quaternionic dimensions from these hypermultiplets in this new phase is $4 \times \mathbf{2}-\operatorname{dim} S U(2)=5$. The effect of this is to completely break $S U(2)$ including any $U(1)$ subgroup. Thus there are $11+5=16$ hypermultiplets and $11-1=10$ vector multiplets in agreement with the type IIB picture above.

In [11] there was some speculation that $Z$ might be some particular complete intersection in a weighted projective space. This was based purely on the Hodge numbers and we will not try to verify this conjecture here.

Both $X$ and $Z$ are members of the set of "Voisin" Calabi-Yau manifolds studied in 35, 36. Consider $\left(\mathrm{K} 3 \times T^{2}\right) / \mathbb{Z}_{2}$ where the involution acts, as before, by inversion on the $T^{2}$ and satisfies (6) on the K3 surface. In general there are many possibilities for the fixed-point set of the action on the K3 surface and they were classified by Nikulin [37]. Essentially the fixed point set usually consists of a set of algebraic curves of which one may have a non-zero genus and the others are all rational. Each possibility leads to different Calabi-Yau manifolds. They are shown in figure 4 . From this figure the Hodge numbers of the resulting manifolds are

$$
\begin{aligned}
& h^{1,1}=5+3 r-2 a \\
& h^{2,1}=65-3 r-2 a,
\end{aligned}
$$

except for a couple of exceptions, one of which is $X$.

Each dot, circle or square in figure 4 represents a different manifold. It was conjectured in [35] and then proven in [31] that pairs related by a reflection in the vertical $r=10$ line are mirror pairs - hence the apparent symmetry of the figure. Those without mirrors are 
shown as squares. See [37] for the difference between dots and circles.

It would be nice to give a model for the space to which the type IIA string on $X$ goes upon the phase transition. Such a space must be the mirror of $Z$. Unfortunately figure $⿴$ shows that $Z$ is one of the spaces which does not have a Voisin mirror. This should not come a complete surprise for the following reason. When we go to the interesting degeneration point in the type IIA moduli space, as we explained earlier, the covering K3 surface must have volume of order the Planck scale. When we go through the phase transition we lose the modulus from the vector multiplet which gave us the ability to change the volume. Therefore it seems reasonable that the space we go to from the phase transition is "stuck" at the Planck scale. A more precise statement of this is that the mirror of $Z$ has no Calabi-Yau phase and is of the type discussed in [38].

The extremal transition we have considered, from $X$ to $Z$, takes us from one Voisin manifold to another. One can certainly envision a generalization of this process where we move to more Voisin manifolds. All we need to do is to further degenerate the quotienting so as to pick up more fixed points. Unfortunately it is not simple to do this within the current context. The moduli space of complex structures on $Z$ is a subset of those on $X$ and it follows that every degeneration of $Z$ can be understood in terms of a degeneration of the covering K3 rather than a degeneration of the quotient. This does not mean it cannot be done however as we have yet to explore the hypermultiplet moduli space.

\section{Remarks}

In this paper we have studied two aspects of type II string theory on $X$, where $X$ which is an orbifold without fixed points of $\mathrm{K} 3 \times T^{2}$. Firstly there are quite subtle discrete degrees of freedom coming from the R-R sector which must be employed to obtain modular invariance of the dual heterotic string. The precise explanation of this effect in terms of the type II string is not yet understood and one might say we have no right to expect we can understand it until we have a better definition for string theory. What we did however was to give an explicit geometrical interpretation of these degrees of freedom.

Secondly we examined a phase transition from the type IIB string on $X$ to another type IIB string theory on $Z$. It turned out that this analysis was intimately connected to the first question of discrete degrees of freedom. These degrees of freedom affect the numbers of massless states precisely at the point of degeneration connecting the two phases. This may give some further insight into these discrete parameters. It is also gives further evidence that we have indeed correctly identified a type II/heterotic dual pair.

Construction of the heterotic dual to the type IIA string on $X$ was obtained by orbifolding the generally-believed string-string duality in six dimensions compactified on a torus. One would have hoped that this case would have been easy as the orbifold action was free but we still had problems with discrete degrees of freedom. In order to understand more dual pairs with $N=2$ supersymmetry in four dimensions it would be nice to extend this construction 
to orbifolds with fixed points. In [7] it was shown that such a process is problematic. There it was stated that "orbifolding does not commute with duality". It would be nice to think that we can rescue orbifolding from such a fate by understanding how it should be modified in the non-perturbative context. The example studied in this paper might give some insight into this problem as $Z$ is constructed from an orbifold with fixed points.

Extremal transitions are performed by shrinking down $S^{2}$ 's, $S^{3}$ 's and/or rational surfaces within a Calabi-Yau manifold to obtain a degenerate space. This is then followed by a reverse procedure to give a new manifold. Since the objects which are shrunk down are all simply connected it appears, at first sight, that $\pi_{1}$ cannot change during such a transition. If this were the case then $\pi_{1}$ would provide an obstruction to connecting up the moduli space of all Calabi-Yau manifolds with extremal transitions. From the example studied in this paper we see that $\pi_{1}$ can change. How this happens is clear from figure 2. The two spheres in this figure within $Y$ are identified by the freely acting $\mathbb{Z}_{2}$ and so we can write a non-contractable loop in $X$ as the image on the quotient of a line connecting one sphere to the other. As we go to the degeneration, $X^{\sharp}$, the spheres approach and so this line shrinks down to zero length, i.e., the loop in $X$ has contracted and $\pi_{1}(Z)=0$. Thus, as the $S^{2}$ 's shrink down, an $S^{1}$ also shrinks down as a side-effect. As $\pi_{1}$ can no longer be viewed as an obstruction, the possibility remains that all Calabi-Yau manifolds may be connected by extremal transitions.

Now that we have identified the phase into which the type II string on $X$ passes it would be nice to do further analysis of the corresponding Seiberg-Witten theory. In [39] it was shown that Seiberg-Witten theory could be recovered from the $\alpha^{\prime} \rightarrow 0$ limit of the dual pair conjectured in [5] (see also [40, 41, 42, 43] for discussions relevent to this). That is, the Yang-Mills theory of the heterotic string theory is linked to the algebraic geometry of the type II theory to agree with [44]. Here such an analysis may prove even more interesting because of the presence of the Higgs phase. One thing to note is the fact that the five new interesting hypermultiplet moduli are identified as blowing-up modes within $Z$. That is, they are Kähler modes and are thus subject to world-sheet instanton corrections. To remove such effects we need to go to the type IIA string on the mirror of $Z$. As explained above, it is probably the case that $Z$ has no Calabi-Yau mirror. This should not discourage us however as one can still build a model for the moduli space of complex structure of the mirror by analyzing it in some other phase, such as a Landau-Ginzburg phase. It is not immediately apparent how to do this as we have not written $Z$ in the form of a complete intersection. Clearly this deserves further attention.

\section{Acknowledgements}

A great deal of this work arose as a side-product from some collaborative work with M. Gross. I thank M. Gross for many important contributions. It is also a pleasure to thank S. Chaudhuri, I. Dolgachev, B. Greene, D. Morrison and A. Strominger for useful conversations as well as the "Plancksters" at the I.T.P., Santa Barbara program where part of this work was 
done under the NSF grant PHY94-07194. The work of the author is supported by a grant from the National Science Foundation.

\section{References}

[1] E. Witten, String Theory Dynamics in Various Dimensions, Nucl. Phys. B443 (1995) 85-126.

[2] C. Hull and P. Townsend, Unity of Superstring Dualities, Nucl. Phys. B438 (1995) 109-137.

[3] M. Duff, Strong/Weak Coupling Duality from the Dual String, Nucl.Phys. B442 (1995) 47-63.

[4] P. S. Aspinwall and D. R. Morrison, U-Duality and Integral Structures, Phys. Lett. 355B (1995) 141-149.

[5] S. Kachru and C. Vafa, Exact Results For N=2 Compactifications of Heterotic Strings, Nucl. Phys. B450 (1995) 69-89.

[6] A.Klemm, W.Lerche, and P.Mayr, K3-Fibrations and Heterotic-Type II String Duality, CERN 1995 preprint CERN-TH/95-165, hep-th/9506112.

[7] C. Vafa and E. Witten, Dual String Pairs With $N=1$ and $N=2$ Supersymmetry in Four Dimensions, Harvard and IAS 1995 preprint HUTP-95/A023, hep-th/9507050.

[8] S. Chaudhuri and J. Polchinski, Moduli Space of CHL Strings, ITP 1995 preprint NSF-ITP95-50, hep-th/9506048.

[9] P. S. Aspinwall, Some Relationships Between Dualities in String Theory, Cornell 1995 preprint CLNS-95/1359, hep-th/9508154.

[10] S. Chaudhuri and D. Lowe, Type IIA-Heterotic Duals with Maximal Supersymmetry, ITP 1995 preprint NSF-ITP-95-76, hep-th/9508144.

[11] S. Ferrara, J. Harvey, A. Strominger, and C. Vafa, Second Quantized Mirror Symmetry, CERN et al 1995 preprint CERN-TH 95/131, hep-th/9505162.

[12] M. Reid, The Moduli Space of 3-folds with $K=0$ May Nevertheless be Irreducible, Math Ann. 278 (1987) 329-334.

[13] P. Green and T. Hübsch, Possible Phase Transitions Among Calabi-Yau Compactifications, Phys. Rev. Lett. 61 (1988) 1163-1166.

[14] A. Strominger, Massless Black Holes and Conifolds in String Theory, Nucl. Phys. B451 (1995) 96-108.

[15] B. R. Greene, D. R. Morrison, and A. Strominger, Black Hole Condensation and the Unification of String Vacua, Nucl. Phys. B451 (1995) 109-120. 
[16] G. Aldazabal, A. Font, L. E. Ibáñez, and F. Quevedo, Chains of N=2, D=4 Heterotic/Type II Duals, CERN et al 1995 preprint CERN-TH/95-270, hep-th/9510093.

[17] P. Candelas, P. Green, and T. Hübsch, Rolling Among Calabi-Yau Vacua, Nucl. Phys. B330 (1990) 49-102.

[18] D. R. Morrison, Through the Looking Glass, Lecture at CIRM conference, Trento (1994), to appear.

[19] C. Vafa and E. Witten, On Orbifolds with Discrete Torsion, J. Geom. Phys. 15 (1995) 189-214.

[20] P. S. Aspinwall and D. R. Morrison, Stable Singularities in String Theory, Cornell and Duke 1995 preprint CLNS-95/1325, DUKE-TH-95-89, hep-th/9503208, appendix by M. Gross, to appear in Commun. Math. Phys.

[21] C. Vafa, Modular Invariance and Discrete Torsion on Orbifolds, Nucl. Phys. B273 (1986) 592-606.

[22] R. Bott and L. W. Tu, Differential Forms in Algebraic Topology, Springer-Verlag, New York, 1982.

[23] P. S. Aspinwall and D. R. Morrison, Chiral Rings Do Not Suffice: N=(2,2) Theories with Nonzero Fundamental Group, Phys. Lett. 334B (1994) 79-86.

[24] J. Milne, Étale Cohomology, Princeton University Press, 1980.

[25] P. S. Aspinwall and D. R. Morrison, String Theory on K3 Surfaces, Duke and IAS 1994 preprint DUK-TH-94-68, IASSNS-HEP-94/23, hep-th/9404151, to appear in "Essays on Mirror Manifolds 2".

[26] K. S. Narain, New Heterotic String Theories in Uncompactified Dimensions < 10, Phys. Lett. 169B (1986) 41-46.

[27] K. S. Narain, M. H. Sarmadi, and C. Vafa, Asymmetric Orbifolds, Nucl. Phys. B288 (1987) $551-577$.

[28] P. S. Aspinwall, Enhanced Gauge Symmetries and K3 Surfaces, Cornell 1995 preprint CLNS95/1348, hep-th/9507012, to appear in Phys. Lett. B.

[29] E. Witten, Some Comments on String Dynamics, IAS 1995 preprint IASSNS-HEP-95-63, hep-th/9507121.

[30] N. Seiberg and E. Witten, Electric - Magnetic Duality, Monopole Condensation, and Confinement in N=2 Supersymmetric Yang-Mills Theory, Nucl. Phys. B426 (1994) 19-52.

[31] P. S. Aspinwall and D. R. Morrison, Mirror Symmetry and the Moduli Space of K3 Surfaces, in preparation.

[32] W. Barth, C. Peters, and A. van de Ven, Compact Complex Surfaces, Springer, 1984. 
[33] D. Morrison, Semistable Degenerations of Enriques' and Hyperelliptic Surfaces, Duke Math. J. 48 (1981) 197-249.

[34] J. Shah, Projective degenerations of Enriques' surfaces, Math. Ann. 256 (1981) 475-495.

[35] C. Voisin, Miroirs et involutions sur les surfaces K3, in A. Beauville et al., editors, "Journées de Géométrie Algébrique d'Orsay", volume 218 of Astérisque, pages 273-323, Société Mathématique de France, 1993.

[36] C. Borcea, K3 Surfaces with Involution and Mirror Pairs of Calabi-Yau Manifolds, Rider College 1994 preprint.

[37] V. V. Nikulin, Discrete Reflection Groups in Lobachevsky Spaces and Algebraic Surfaces, in "Proceedings of the International Congress of Mathematicians, Berkeley", pages 654-671, 1986.

[38] P. S. Aspinwall and B. R. Greene, On the Geometric Interpretation of $N=2$ Superconformal Theories, Nucl. Phys. B437 (1995) 205-230.

[39] S. Kachru et al., Nonperturbative Results on the Point Particle Limit of N=2 Heterotic String, Harvard 1995 preprint HUTP-95/A032, hep-th/9508155.

[40] M. Billó et al., A Search for Non-Perturbative Dualities of Local N=2 Yang-Mills Theories from Calabi-Yau Threefolds, CERN et al 1995 preprint CERN-TH 95/140, hep-th/9506075.

[41] V. Kaplunovsky, J. Louis, and S. Theisen, Aspects of Duality in N=2 String Vacua, Munich 1995 preprint LMU-TPW 95-9, hep-th/9506110.

[42] G. L. Cardoso, D. Lüst, and T. Mohaupt, Non-Perturbative Monodromies in N=2 Heterotic String Vacua, Humboldt 1995 preprint HUB-IEP-95/12, hep-th/9507113.

[43] I. Antoniadis and H. Partouche, Exact Monodromy Group of N=2 Heterotic Superstring, École Polytechnique 1995 preprint CPTH-RR370.0895, hep-th/9509009.

[44] N. Seiberg and E. Witten, Monopoles, Duality and Chiral Symmetry Breaking in N=2 Supersymmetric QCD, Nucl. Phys. B431 (1994) 484-550. 